\title{
Literature Review of Machine Vision in Application Field
}

\author{
ChenZhang $^{1 *}$, XuewuXu ${ }^{1,2}$, ChenFan $^{1}$, GuopingWang ${ }^{1}$ \\ ${ }^{1}$ Mechanical Engineering, Xi'an Jiaotong University City College, Xi'an, Shaanxi Province, 710018, P. R. China \\ ${ }^{2}$ Xi'an Jiaotong University Intelligent Robot Innovation Institute, Xi'an, Shaanxi Province, 710018, P. R. China
}

\begin{abstract}
Aiming at the application and research of machine vision, a comprehensive and detailed elaboration is carried out in its two application areas: visual inspection and robot vision. Introduce the composition, characteristics and application advantages of the machine vision system. Based on the analysis of the current research status at home and abroad, the application development trend of machine vision is prospected.
\end{abstract}

\section{Introduction}

Recent years, with the global "Industry 4.0", "Made in China 2025" and other market backgrounds, machine vision as the core technology of artificial intelligence has been rapidly developed in China, and its application scope covers industry, agriculture, medicine, Military, aerospace, scientific research and other fields of the national economy, as China has become a global manufacturing processing center, high requirements for parts processing and related advanced production lines have made many international advanced machine vision systems and application experiences penetrate into China . Machine vision has been developed from scratch for 20 years. Its functions and application scope have been continuously improved and matured with the development of industrial automation. Machine vision emphasizes practicality. It must be able to adapt to the harsh environment of the industrial site and reasonable cost performance, higher fault tolerance and safety, stronger real-time, high precision and high speed, and has the advantages of non-contact, so it has Wide application prospects.

\section{Machine vision system and its characteristics}

$80 \%$ of the information that humans know and understand the external world comes from vision, while machine vision uses machines to replace human eyes for measurement and judgment ${ }^{[1]}$. The ultimate goal of machine vision is to make the computer more intelligent, make it look like a human, observe and understand the world through vision, and then analyze and make decisions while having the ability to adapt to the environment independently ${ }^{[2]}$. Since machine vision is to establish an artificial intelligence system that obtains "useful information" from images or multi-dimensional data by studying related theories and technologies, it is a branch of artificial intelligence and a comprehensive technology that includes images Processing, mechanical engineering, control, electric light source lighting, optical imaging, sensor technology, analog and digital video technology, computer software and hardware technology and human-machine interface technology, etc., these technologies can work together to form a complete machine vision application system, A typical industrial machine vision system is shown in Figure 1, including: light source, lens (fixed focus lens, zoom lens, telecentric lens, microscope lens), camera (including CCD camera and COMS camera), image processing unit ( Or image capture card), image processing software, monitor, communication/input output unit, etc ${ }^{[3]}$. 


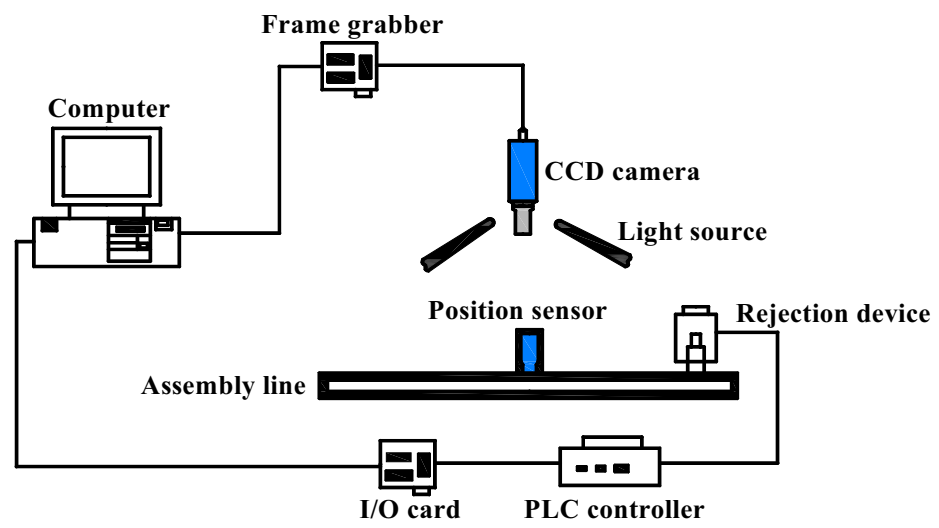

Figure 1. Industrial machine vision system

The most basic characteristics of machine vision systems are practicality, versatility and real-time ${ }^{[4]}$. That is to improve the flexibility and automation of production. In some dangerous environments that are not suitable for manual operations or where long-term artificial vision is difficult to meet the requirements, machine vision is usually used to replace artificial vision. In addition, in large-scale repetitive production processes Among them, because artificial vision cannot work continuously, it will cause fatigue and increase the error of workpiece production. The method of detecting with machine vision can greatly improve the efficiency, accuracy, and automation of production.

\section{Domestic and foreign research areas of machine vision}

The rapid development of machine vision in recent years stems from the rapid improvement of the computer industry level and the country's vigorous development of

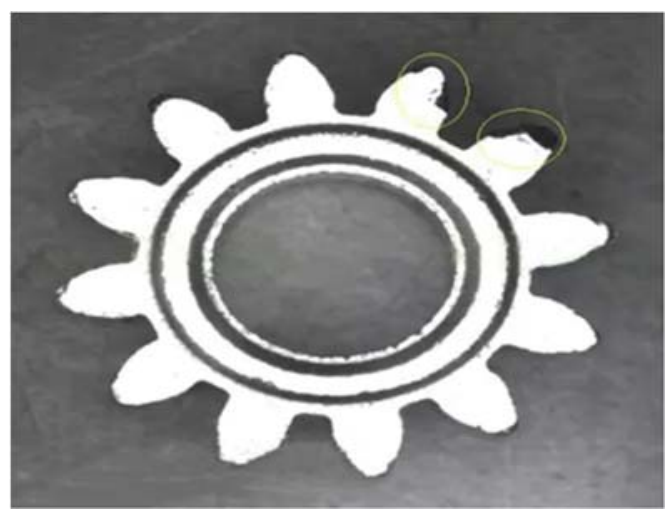

Figure 2. spray gun model new disciplines such as artificial intelligence and big data, which has made the machine vision system continuously improved and the degree of practicality has been further improved. For example: a wide variety of light sources, optical lenses that can automatically adjust the aperture and zoom, advanced vision sensors, 3D stereo cameras, and computers.

At present, scholars at home and abroad can be roughly divided into two fields according to the application direction of machine vision: one is the detection field, and the other is the robot vision field ${ }^{[5]}$. The former is divided into high-precision quantitative detection and qualitative or semi-quantitative detection without a meter. It is mainly used in gear tooth defect detection, aviation radiator positioning drilling accuracy detection, traffic control vehicle detection, character recognition, etc. See Figure 2, 3, 4, 5, etc. The latter is used to guide the robot's operations and actions in a wide range, such as picking out the required workpieces from the messy workpieces and placing them in a suitable position.

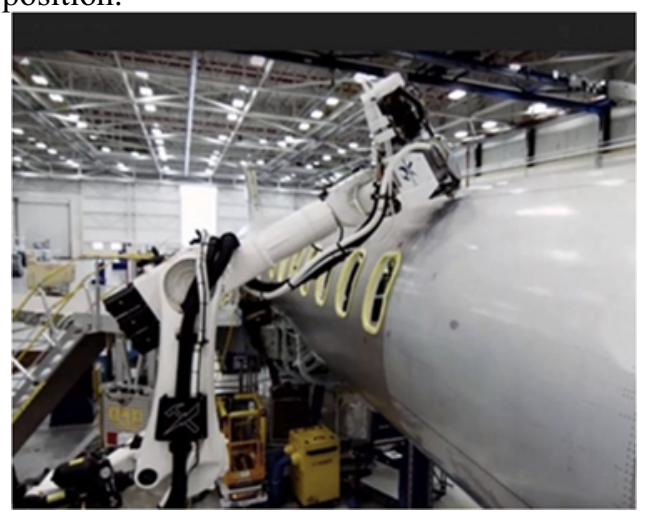

Figure 3. Aviation radiator positioning drilling accuracy detection 


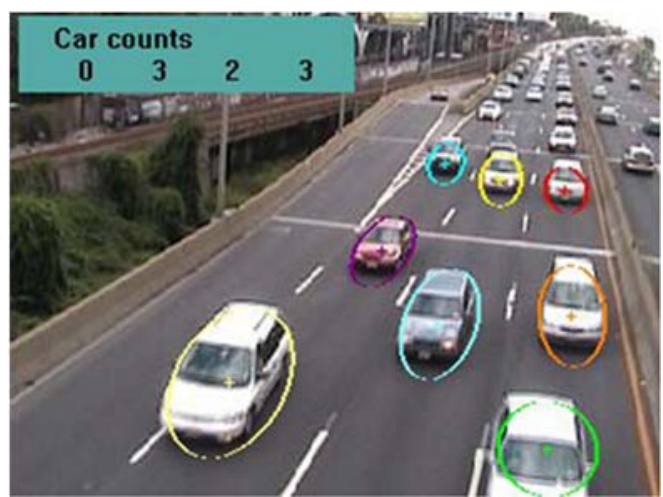

Figure 4. traffic control vehicle detection

From the perspective of application, the research of machine vision includes automatic detection and recognition of workpieces ${ }^{[6]}$, automatic classification of food $^{[7]}$, autonomous navigation and obstacle avoidance of unmanned vehicles ${ }^{[8]}$, forest fire monitoring and flooding areas Surveillance ${ }^{[9]}$, as well as feature extraction of shoe textures based on machine vision, robots to draw lines and glue the appearance of shoes, deep learning and neural network algorithms based on machine vision ${ }^{[10]}$ and so on. From the perspective of the processing process, machine vision is divided into two stages: low-level vision and high-level vision. The bottom level includes edge detection, feature extraction, image segmentation, etc., and high-level vision includes feature matching, threedimensional modeling, scene analysis and understanding ${ }^{[11]}$.

From a methodological perspective, machine vision can also be divided into active and passive. The difference between the two is that active is not easily affected by ambient light, which means that it can understand and adapt to the environment more effectively ${ }^{[12]}$. In addition, there are methods based on features and models, both of which have advantages and disadvantages.

From an overall point of view, machine vision is sometimes called computer vision. This is because the research of the two is generally similar. If subdivided, the difference between the two is that computer vision is widely used in the field of robotics. Machine vision is more widely used in the field of industrial inspection. In addition, computer vision focuses more on the academic research level, while for machine vision, it focuses more on the application level. Machine vision needs to consider complex issues such as noise, lighting, occlusion, and so on. At the same time, it also needs to consider effective image processing, such as noise reduction and smoothing.

Wei Xiaoling of Hebei University of Technology and others have used machine vision to detect gear teeth in response to the traditional manual methods for detecting gear defects with low efficiency and low precision. The author proposed a method for making image templates to achieve the Gear teeth segmentation, and for the wear detection problem of a single gear tooth, the area of the gear tooth and the circumference of the gear tooth are respectively used as the basis for judging the wear of a single gear tooth. Finally, the image of the collected gear is denoised and imaged by the computer. Relevant image processing such as enhancement, image binarization,

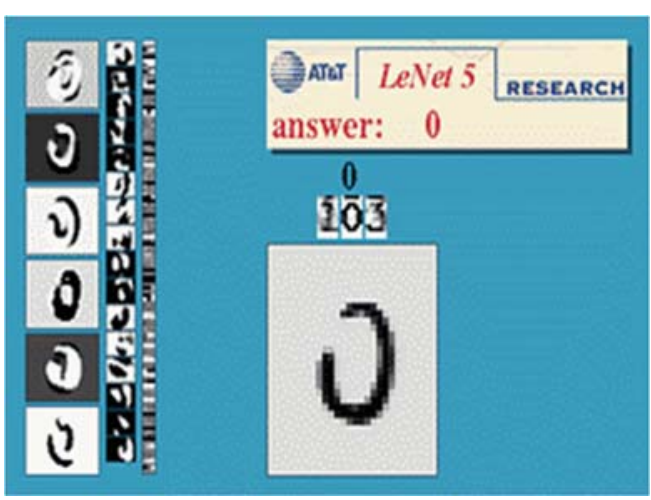

Figure 5. character recognition

image morphology, image edge feature extraction, etc. The experimental results show that the machine vision method is used to detect gear defects, which can accurately identify defective gear teeth and achieve better results $^{[13]}$.

Fan Yunlei and others of Jiangnan University designed a sugarcane cutting equipment based on machine vision based on machine vision technology and PLC control technology in response to the current problem of high sugarcane planting and cutting bud rate. The equipment uses rubber rollers to hold the sugarcane for transportation. The camera collects the sugarcane image and recognizes the stem section. By offsetting the position of the stem section, the position of the cutting point is obtained; the upper computer sends the position of the cutting point to the PLC through TCP communication., PLC completes the positioning and cutting action of the cutting point by controlling the cutting parts and rubber roller parts. The distance between the cutting point and the stem section can be adjusted. The results show that the equipment can achieve high cutting precision and low bud damage rate. Vision software The recognition rate of stem nodes is $95.5 \%$, the rate of cutting seedlings is zero, and the cutting error is within $8 \mathrm{~mm}^{[14]}$.

Vasiliy N. Kruglov of Ural Federal University aimed at the problems of manual sampling method for evaluating the particle size of crushed materials, the complexity of measurement and many negative factors, and the inability to conduct objective measurement with sufficient frequency. The author proposed a development control based on machine vision. The system analyzes a possible algorithm for boundary detection of broken mineral particles using convolutional neural networks, as shown in Figure 6. Gives the structure of the neural network, introduces the construction of ore particle image training and test data sets, and studies the improved underlying neural network. During the research process, it is found that the basic model has the best quality of identifying boundary points. It shows an accuracy of $83 \%$ of the target class prediction. Based on the boundary drawn on the test image, it can be concluded that the convolutional neural network can correctly identify boundary points with high probability. In the absence of boundaries, it rarely makes mistakes, but it often makes mistakes when identifying true boundary points. The boundary interruption is too large to be closed using morphological operations on the binarization mask. However, using the "watershed" 
algorithm will reduce the recognition error of boundary

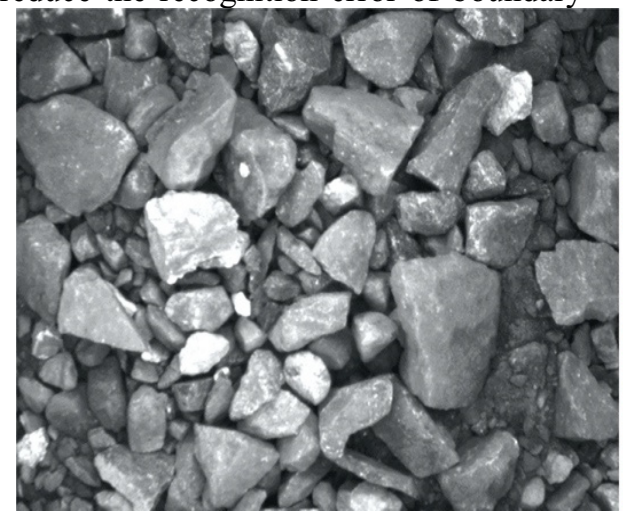

points $^{[15]}$.

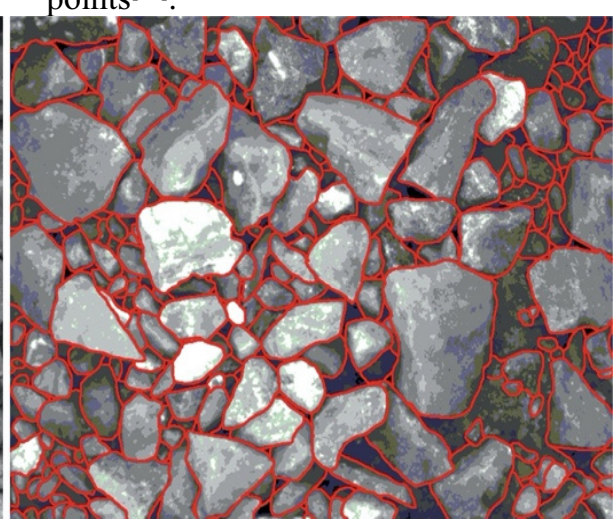

Figure 6. Original images and marked images

Dan Berco of Nanyang Technological University and others aimed at the machine vision system's excessive reliance on cameras and processor modules, making this layout cumbersome and inefficient in terms of speed and power consumption. The author established an operating system that can simulate biological eyes. And the independent platform function equipment, the next evolutionary step for machine vision perception, a bionic, hybrid electrophysiological response neuron device is proposed, which simulates the combined function of cone cells and bipolar cells in the retina, Experimental results show that this artificial cognitive retina (that is, thinking eyes) completes image recognition tasks faster than machine vision software algorithms ${ }^{[16]}$.

\section{Conclusion}

With the continuous development of artificial intelligence technology, the more advanced the types of vision sensors in machine vision, the more and more machine vision functions. Under the current situation, the application of machine vision in automated industrial production still occupies a pivotal position, because machine vision systems can Quickly obtain a large amount of information, so it is widely used in many inspection equipment, which can improve the efficiency of production parts, and can also be applied to the maturity inspection of agricultural and forestry crops. However, machine vision technology is more complicated, and foreign scholars continue to use bionic vision Equipment to simulate the eyes of animals or humans, with the development potential of machine vision technology, it is believed that in the near future, it will be more and more widely used in modern and future manufacturing enterprises.

\section{Acknowledgments}

This work was financially supported by the Special scientific research project of Shaanxi Provincial Department of Education (Program No.20JK0742) and Xi'an Jiaotong University City College Research Fund Project (Project No.2019Q06).

\section{References}

1. Janusz S, Pawel H. Workspace supervising system for material handling devices with machine vision assistance [J]. Journal of KONBiN, 2009 (11/12): 716.

2. Ramesh C.Jain, Rangachar Kasturi, Brian G. Machine Vision [M]. China Machinery Press; McGraw Hill Education (Asia), 2003.

3. Zhao Peng. Research and development of machine vision [M]. Beijing: Science Press, 2012.

4. Ouyang Zhi, Xiao Xu. Application of machine vision in intelligent manufacturing $[\mathrm{J}]$. The era of big data, 2018 (03): 9-12.

5. Xie Jianbin, etc. 20 Lectures on Visual Machine Learning [D]. Beijing: Tsinghua University Press, 2015.

6. Feng $\mathrm{Xi}$, Wu Jingjing, An Wei. Automatic measurement system for large-size workpieces based on machine vision[J]. Sensors and Microsystems, 2019, 38(04): 104-107.

7. Yan Zugen, Li Ming, Xu Kefei, et al. Research on machine vision technology of high-speed robotic sorting system [J]. Packaging and Food Machinery, 2014, 32(1): 28-31.

8. Zeng Shifeng, Wu Jinjun, Ye Zhiwen, Ye Miaoxin, Lai Yiwen, Ding Fan. Autonomous driving system design based on machine vision [J]. Electronic World, 2020(05): 197.

9. Liu Shudong, Yao Wenbo, Zhang Yan. Forest fire monitoring based on machine vision in foggy conditions [J]. Computer Engineering and Science, 2020, 42(07): 1253-1261.

10. Xu Hui, Zhu Yuhua, Zeng Tong, Li Zhihui. A review of image semantic segmentation methods using deep neural networks [J/OL]. Computer Science and Exploration: $\quad 1-21$ [2020-09-17]. http://kns.cnki .net/kcms/detail/11.5602.TP.2020090 3.1737.010.html.

11. Sun Zhijie, Xu Hongli. A mapping method from lowlevel visual features to high-level semantics of an 
image[J]. Computer Applications, 2004(12): 22-24.

12. Arabnia Hamid R,Deligiannidis Leonidas, Tinetti Fernando G. Image Processing, Computer Vision, and Pattern Recognition[M].CSREA (MLI):202003-17.

13. Wei Xiaoling, Cui Yue, Wang Xiaopeng. Research on tooth defect detection based on machine vision[J]. Coal Mine Machinery, 2020, 41(09): 35-37.

14. Fan Yunlei, Zhou Deqiang, Deng Qianran, He Fengguang, Wang Meili. Design and experiment of sugarcane cutting equipment based on machine vision[J]. Research on Agricultural Mechanization, 2021, 43(05): 55-60.

15. Kruglov V N. Using Open Source Libraries in the Development of Control Systems Based on Machine Vision[C]//IFIP International Conference on Open Source Systems. Springer, Cham, 2020: 70-77.

16. Berco D, Ang D S, Zhang H Z. An Optoneuronic Device with Realistic Retinal Expressions for Bioinspired Machine Vision[J]. Advanced Intelligent Systems, 2020, 2(2): 1900115. 